\title{
Teaching Reform and Practice of the Course of Analytical Instrument for Agriculture
}

\author{
Weiyu Zhang \\ College of Engineering and Technology \\ Tianjin Agricultural University \\ Tianjin, China \\ 525156985@qq.com \\ Hua Liu \\ College of Engineering and Technology \\ Tianjin Agricultural University \\ Tianjin, China \\ 41599386@qq.com
}

\author{
Jinfeng Dong \\ College of Engineering and Technology \\ Tianjin Agricultural University \\ Tianjin, China \\ 873980627@qq.com \\ Yu Shi \\ College of Engineering and Technology \\ Tianjin Agricultural University \\ Tianjin, China \\ syych83@tjau.edu.cn
}

\begin{abstract}
According to today's social enterprises with the characteristics of the demand, according to the agricultural instrument analysis course, requirements for the application type undergraduate engineering practical ability, combined with the agricultural analysis instrument course teaching, there are a series of problems in teaching, to agriculture analysis instrument and teaching method reform and students' engineering application ability cultivation research. The train of thought, content and method of the teaching reform of agricultural analytical instruments are explored and practiced. Practice has proved that through strengthening the practice teaching, can cultivate students' engineering ability and innovation ability to adapt to the social demand.
\end{abstract}

Keywords-demand; analysis instrument; project-driven; creative ability

\section{LITERATURE REVIEW}

To cultivate high quality talents with innovative consciousness and innovative ability is the theme of higher education reform, to strengthen experimental teaching and to cultivate students' ability has become a common understanding in the teaching of higher education in China. Experimental teaching is not only an important link between theory and practice, but also an important way to cultivate students' ability, innovative consciousness and comprehensive quality. In addition to playing an important role in university research and service, large scale instruments play an important role in experimental teaching in universities, which is an indispensable teaching resource in Colleges and universities. Agricultural analytical instrument is an indispensable condition for the analysis of the experiment teaching of instrumental analysis[1]. In recent years, with the school of scientific research and teaching funds continue to invest, the school of large-scale equipment, regardless of the quantity or quality has been greatly improved. While improving the level of scientific research and analysis of testing services, expanding the scope and precision of analysis, the combination of experimental teaching and modern science and technology has been greatly improved. Also due to constraints of large-scale instruments are expensive, instrument less units, with complicated operation and experimental time is limited, many factors, students in the experimental teaching of large-scale instruments and equipment by greatly restricted[2].

At present, the main mode of large-scale instruments and experimental teaching: demonstration teaching, mainly in the experimental demonstration, pay attention to the theory of knowledge rather than the mastery of skills; II virtual teaching, virtual software as a platform for simulation operation; third, open experimental teaching, to the student actual operation. From the effect of teaching, only the open experimental teaching is the best[3].

Agricultural analytical instruments are mainly used for the qualitative and quantitative analysis of the object to be measured in the practical application. These tasks can be achieved through "UV visible absorption spectroscopy,", "infrared absorption spectroscopy,", "atomic absorption spectrometric method, gas chromatographic analysis method", "high performance liquid chromatographic analysis method and other means to achieve. Agricultural College and professional students to learn the purpose of the course is: the students on the basis of differences in the physical properties of each component in the mixture to be measured, choose suitable analytical instrument and analytical methods of qualitative analysis and quantitative analysis, establish accurate qualitative and quantitative concepts and master and the related basic theory, principle and experimental technique of determination, to lay a good foundation for subsequent high-tech agricultural scientific research work[4].

\section{TRADITIONAL TEACHING}

At present, the main mode of large-scale instruments and experimental teaching: demonstration teaching, mainly in the experimental demonstration, pay attention to the theory of knowledge rather than the mastery of skills; II virtual teaching, virtual software as a platform for simulation operation; third, 
open experimental teaching, to the student actual operation. From the effect of teaching, only the open experimental teaching is the best[5].

Agricultural analytical instruments are mainly used for the qualitative and quantitative analysis of the object to be measured in the practical application. These tasks can be achieved through "UV visible absorption spectroscopy", "infrared absorption spectroscopy", "atomic absorption spectrometric method, gas chromatographic analysis method", "high performance liquid chromatographic analysis method and other means to achieve. Agricultural College and professional students to learn the purpose of the course is: the students on the basis of differences in the physical properties of each component in the mixture to be measured, choose suitable analytical instrument and analytical methods of qualitative analysis and quantitative analysis, establish accurate qualitative and quantitative concepts and master and the related basic theory, principle and experimental technique of determination, to lay a good foundation for subsequent high-tech agricultural scientific research work.

\section{TEACHING METHOD}

\section{A. The Teaching Content of Practical and Forward-Looking}

Succinctly theories and methods of agricultural instruments, in the actual teaching in the latest research results and problems into teaching content, allowing the development of cuttingedge content of classroom teaching subjects followed, deepen students' understanding and awareness programs. In order to enhance students' cognitive ability and time, the program is divided into four parts:

- Cognitive theory analytical instruments

- The use of sophisticated analytical instruments

- Waste instruments disassembly

- Used Precision measuring instruments

\section{B. A Variety of Teaching Methods}

Combined with a relatively small number of instruments agricultural analysis features, combined with multimedia teaching, so that students not in contact with the instrument before the instrument has a perceptual awareness, learning laboratory for students to enter and lay a good foundation. By grouping students into the laboratory study, so that students into the laboratory analytical instruments recognize objects, basic operation training and practice teaching basic analytical applications. Arrange over the guidance of teachers, the use of teachers do not move; press the group of students to complete a cycle three experiments.

\section{Establishing Practice Teaching System}

Establish a "course - Experiment - Practice - Innovation" Practice Teaching System. Experimental Course strengthen students' analytical instruments "basic skills training; practice teacher arranged for students to participate in research projects, to meet the students' research, autonomous learning needs; cultivate students' creative thinking and practical ability.
Engineering and Technical College of Agricultural Electrification and Automation relies on experimental teaching demonstration center, the training objectives and targeting schools combine to improve their overall quality, ability and innovation as the fundamental, continue to strengthen the investment and construction of experimental teaching base. New Innovation Lab, including UV spectrophotometer, infrared spectrometer, fluorescence spectrometer and other large equipment ensure that all aspects of the practice of teaching. Undergraduate students under the guidance of the teacher's guidance, combined with the subject to enter the laboratory for scientific experiments.

\section{Methods}

Teaching philosophy developed a design-oriented, capacity-building project training program targeting the Practical Teaching Mechatronics System Design course is divided into research projects, curriculum and course design three experimental projects based in part on educational practice. Through "experimental course curriculum project + curriculum + design" Three Links closely integrated practice teaching, and enhance students' perceptions of mechanical and electrical engineering, master the basic experimental methods, to master the basic skills of mechatronic systems knowledge to further deepen the curriculum understanding of content, improve the ability to analyze and solve practical engineering problems.

In terms of teaching methods and means, so that the "three combinations": First, the theory and practice teaching combined. Improve the overall ability to learn and apply knowledge, attention to the cultivation of students' self-learning ability; Second, curricular teaching and extracurricular teaching combined. Both curricular emphasis on the quality of teaching, but also to the practice of teaching college students to carry out scientific and technological activities; third is the traditional mode of teaching and modern teaching methods combined. The use of multimedia, CAI courseware and network platform mobilizes the enthusiasm of student learning, develops students' ability. Specifically, the following:

- Through the preparation of teaching materials, combined with professional features, conscious content and teaching to enhance students' logical thinking ability and practical ability and training.

- Fostering awareness of application capabilities and applications in teaching. Usually in teaching, in addition to teaching textbook knowledge, but also the agricultural environment, food safety and other people's livelihood, major events and taught analytical instrument detection technology together with the current, and guide students to think and to deepen awareness of the culture applications. Waste instruments disassembly

- Teachers in the classroom, in addition to using a variety of methods teaching method, demonstration method, tutoring, etc., but also the use of teaching methods in the classroom discussion, ask questions, ask students to think, discuss, and to allow students to come to the blackboard and explain completion, the classroom of 
students to express their views, active classroom atmosphere, but also inspired a keen interest in student learning, to achieve a good teaching effect.

- Analytical instruments for construction and measuring principle characteristics of the production of the corresponding flash animation to optimize the multimedia courseware. These flash animation and video from the instrument to the principle of actual test operations are carried out a detailed description of the dull, obscure instrument knowledge vivid unfolded. Through these diverse courseware, not only increasing the interesting classroom teaching, but also to enable students to master knowledge related instruments.

- In theoretical classroom teaching, curriculum content taught in groups according to dismantling scrap instrument corresponding components, with the kind of explain. This will not only enable students to understand the principle and structure analysis instrument from the rational, but also from the figurative sensibility, a more intuitive understanding of knowledge and a deeper impression, to overcome the sense of distance in the past to rely on language teaching and pictures bring awareness to mobilize the enthusiasm of the students, improve learning efficiency.

- Open active teaching method in teaching, developed excellent course resources platform, this course syllabus, courseware, teaching resources and bibliography, as well as some exercise equipment job forefront of the development of the curriculum related to life on instrument little knowledge of continuous improvement and uploaded to the internet, students can review and prep courses related content on the platform, and you can send your question to the job opening exercises and teacher dedicated mailbox realized teacher-student interaction. At the same time, expanding the teaching space extended teaching time.

\section{OUTCOMES}

\section{A. Student Achievements}

Students interested in the experiment, significantly increased interest in scientific research; students' basic knowledge and basic skills in experimental solid, practical and creative abilities to be strengthened and made some experimental innovations. Experimental Teaching Center significantly, train a large number of both ability and integrity of outstanding students, they declare in the undergraduate college students innovation and entrepreneurship project involved a number of high-quality papers published, participation declared national patents. Since the center was established, I was relying on Experimental Center in Undergraduate journals published more than 48 papers; innovation and entrepreneurship students allowed to project a national training program, the municipal college students innovation and entrepreneurship training programs and university college students innovation and entrepreneurship training program 86. Competition in the innovative design, electronic design competitions and various competitions also gratifying achievements, has won the "Challenge Cup" College Students in extracurricular work competition awards three, Tianjin Undergraduate Electronic Design Contest and the National Electronic Design Contest awards Tianjin Division 3 passengers, Robotics competition awards 15 people in five provinces of North China, Tianjin innovation scholarship 2 people like.

\section{B. Teaching Achievements}

In school support, through continuous efforts experimental center, in the original basis of recent years, have purchased a large number of laboratory equipment, especially the US company PerkinElmer |testing equipment, and gradually formed a PerkinElmer |gathered in Tianjin company spectroscopy apparatus to enhance the integration of experimental teaching equipment, comprehensive. Another Shimadzu, FLUKE and other companies are also involved in our teaching practice and maintenance of precision instruments and equipment and give great support.

\section{CONCLUSION}

This topic based on working process of the precision instrument module teaching mode, practice system from 2008 began to complete practice in mechatronic specialty and achieved remarkable results, has higher theoretical significance and practical value.

- Emphasizes the heuristic teaching, discovery learning and interactive cooperative learning, fully utilize modern educational technology, and emphasize the students' initiative and individual development.

- To change the traditional teaching in the examination as the center based on the knowledge of the degree of evaluation, with scientific knowledge as the foundation, the application of innovation as the goal, practical ability to focus on a variety of assessment methods.

- Adhere to the "to task as the main line, take the teacher as the leadership, take the student as the subject" teaching method, the teaching mode of "teaching, learning and doing" integration task drive, and create a play a subjective initiative, positive enterprising, fully display and development of loose practice teaching environment is conducive to students.

- Make full use of secondary resources to provide students with access to high precision testing equipment, the opportunity to disassemble and maintain. Let students in practice to understand the use of the instrument and the internal structure, more understanding of domestic and international large-scale enterprise instrument circuit design methods and ideas, for students to obtain employment and further study to provide better conditions.

Disassembly and assembly at home and abroad well-known large-scale precision instruments. At the same time, the establishment of disassembly and assembly of the instrument and system maintenance, for students to close contact with a large precision instruments laid the foundation, the theory and 
practice of measurement and control technology and instrument specialty courses opened the a new space, but also to the employment of students develop the channel.

\section{ACKNOWLEDGMENT}

This work was financially supported by the Educational Reform project for the Experimental Teaching Center of Tianjin Agricultural University (2015SY016) and Science \& Technology Correspondent Project of Tianjin (15JCTPJC58600)

\section{REFERENCES}

[1] Huang Jiu Jiu, Wang Sheng bin, Gong Hui. Modern biotechnology experimental teaching center construction practice and thinking [J] Laboratory science, 2014,02:180-183+187.Zhou Yun, Ying Jin-xian,

[2] Yu Zhongan, Zhang Zhenli. To strengthen the construction of experimental teaching demonstration center to improve the quality of practice teaching $[\mathrm{J}]$. Journal of Jiangxi University of Science and Technology, 2010,06:49-52.

[3] LI Xun-ru. Research on practical training equipment of medical equipments maintenance major[J]. China Medical Equipment, $2013,10(10): 30-32$.

[4] Zhang Si-chun, etc..Application of Large-scale Laboratory Instruments and Equipment in Cultivation of Creative Talents in Universities [J]. Research and Explortion in Laboratory 2011, 30(11): 274-277.

[5] Xu Zhi-shan. Culture of The Second Class Construction and Engineering Students* Innovative[D]. Fujian Normal University 2015 